\title{
The Morphoelectrotonic Transform: A Graphical Approach to Dendritic Function
}

\author{
Anthony M. Zador, ${ }^{1}$ Hagai Agmon-Snir, ${ }^{2}$ and Idan Segev ${ }^{2}$ \\ 'Salk Institute, San Diego, California 92037, and 'Department of Neurobiology and Center for Neural Computation, \\ Institute of Life Sciences, Hebrew University, Jerusalem, Israel 91904
}

\begin{abstract}
Electrotonic structure of dendrites plays a critical role in neuronal computation and plasticity. In this article we develop two novel measures of electrotonic structure that describe intraneuronal signaling in dendrites of arbitrary geometry. The log-attenuation $L_{i j}$ measures the efficacy, and the propagation delay $P_{i j}$ the speed, of signal transfer between any two points $i$ and $j$. These measures are additive, in the sense that if $j$ lies between $i$ and $k$, the total distance $L_{i k}$ is just the sum of the partial distances: $L_{i k}=L_{i j}+L_{i k}$ and similarly $\boldsymbol{P}_{i k}=\boldsymbol{P}_{i j}+\boldsymbol{P}_{j k}$. This property serves as the basis for the morphoelectrotonic transform (MET), a graphical mapping from morphological into electrotonic space. In a MET, either $P_{i j}$ or $L_{i j}$ replace anatomical distance as the fundamental unit and so provide direct functional measures of intraneuronal signaling. The analysis holds for arbitrary transient signals, even those generated by nonlinear conductance changes underlying both synaptic and action potentials. Depending on input location and the measure of interest, a single neuron admits many METs, each emphasizing different functional consequences of the dendritic electrotonic structure. Using a single layer 5 cortical pyramidal neuron, we illustrate a collection of METs that lead to a deeper understanding of the electrical behavior of its dendritic tree. We then compare this cortical cell to representative neurons from other brain regions (cortical layer 2/3 pyramidal, region CA1 hippocampal pyramidal, and cerebellar Purkinje). Finally, we apply the MET to electrical signaling in dendritic spines, and extend this analysis to calcium signaling within spines. Our results demonstrate that the MET provides a powerful tool for obtaining a rapid and intuitive grasp of the functional properties of dendritic trees.
\end{abstract}

[Key words: computer models, electrotonic structure, dendritic computation, cable theory, calcium dynamics, dendritic spines, neuronal simulation, dendritic morphology]

Electrotonic structure plays a critical role in neuronal information processing, and in the experimental analyses of neuronal events (Rall, 1959; Rall, 1967; Barrett and Crill, 1974; Jack et

\footnotetext{
Received Mar. 14, 1994; revised Aug. 23, 1994; accepted Sept. 1, 1994.

We thank David Amaral, Norio Ishizuka, Rodney Douglas, and Moshe Rapp for making their reconstructed neurons available, and Christof Koch for many useful discussions. A.M.Z. acknowledges encouragement from Tom Brown during preliminary stages of this work. A.M.Z. was supported by grants from the HSPFO, and by grants to Christof Koch from the ONR and the NIMH Center for Cellular and Molecular Signaling at Caltech. I.S. was supported by the ONR and BSF.

Correspondence should be addressed to Anthony M. Zador, The Salk Institute MNL/S, 10010 North Torrey Pines Road, La Jolla, CA 92037.

Copyright (C) 1995 Society for Neuroscience $0270-6474 / 95 / 151669-14 \$ 05.00 / 0$
}

al., 1983; reviewed in Rall et al., 1992). It is a key determinant of the spatio-temporal integration of synaptic inputs upon the Iree (reviewed in Rall, 1989; Segev et al., 1994), and the same equations also govern chemical signaling within the tree (Zador and Koch, 1994; M. Siegel, E. Marder, and L. Abbot, unpublished observations). While there is growing recognition of the importance of nonlinear, voltage-dependent, dendritic properties (Miyakawa et al., 1992; Regehr et al., 1992; Stuart and Sakmann, 1994; reviewed in Mel, 1994), any understanding of the nonlinear case must begin with the linear case. This was the reason for the elaboration of cable theory (Rall, 1977; Tuckwell, 1989), which over the last 30 years has provided major insights into dendritic function (McKenna et al., 1992). Yet in spite of its importance, many neuroscientists tend to neglect the detailed electrotonic structure of dendrites. In most cases the first-and often only-question posed about the electrotonic structure of a neuron is "What is its electrotonic length, L?" or "How electrically compact is it?"'. However, knowledge of $L$ alone offers very limited understanding since it provides an estimate only of voltage attenuation from the soma. It does not reflect the actual electrotonic structure of the tree as seen by the dendritic sylapses, nor does it give insight into important questions of signal delay and input synchronization at different regions of the dendritic tree.

To elucidate the functional significance of the dendrites, we have developed a simple graphical approach that offers an immediate intuitive understanding of electrotonic structure. The approach is based on two novel measures of dendritic electrotonic structure, the $\log$-attenuation $L_{i j}$ and the propagation delay $P_{i j}$. The log-attenuation (Zador et al., 1991) can be considered a generalization of the standard electrotonic distance $X$ for passive dendrites with arbitrary branching structure. Only for infinite cylinders is $L_{i j}$ equal to the electrotonic distance $X$; in other structures it is a functional measure of the efficacy of signal transfer between any two points $i$ and $j$. The propagation delay $P_{i j}$ is a measure of the rate at which transient signals propagate through the tree (Agmon-Snir and Segev, 1993). 'These measures are developed within the framework of a passive dendritic tree, but they are directly applicable even to the brief localized nonlinear events - such as synaptic and action potentials- that are of greatest interest to the neurobiologist. The power of these measures is that they offer direct and easily interpretable insights into intradendritic signaling, even in complicated dendritic structures.

We use these measures as the basis for a set of graphical transformations, or mappings, of morphological structure into electrotonic structure. These morphoelectrotonic transformations 
Figure $1 . \quad L_{i j}$ and $P_{i j}$ measure signal transfer. In this schematic neuron, a transient current $I_{\text {in }}$ is injected into a branch at location $i$. In $A$, the shaded regions represent the area under the voltage response recorded at the point of injection $i$ and at a recording point $j$. The $\log$-attenuation $L_{i j}=\log \left(V_{i} / \tilde{V}_{j}\right)$ is the logarithm of the ratio of the areas under the two voltage responses. The same attenuation is obtained from the steady-state voltages between these two points. In $B$, the centroids $\hat{t}_{i}^{V}$ and $t_{j}^{v}$ of the same responses are denoted by vertical lines at 1.5 and $2.5 \mathrm{msec}$. The propagation delay $P_{i j}=t_{j}^{V}-t_{i}^{V}$ is the 1.0 msec difference between the centroids.

Definitions of $L_{i j}$ and $P_{i j}$

A

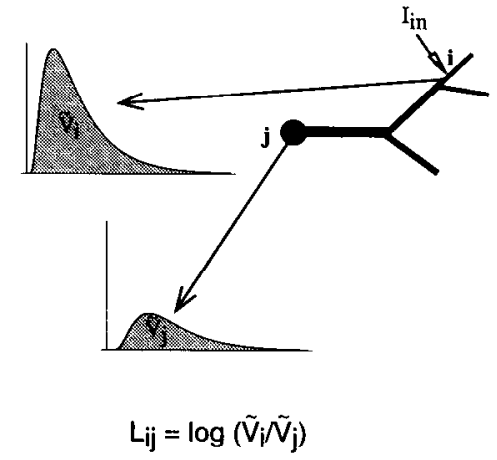

B

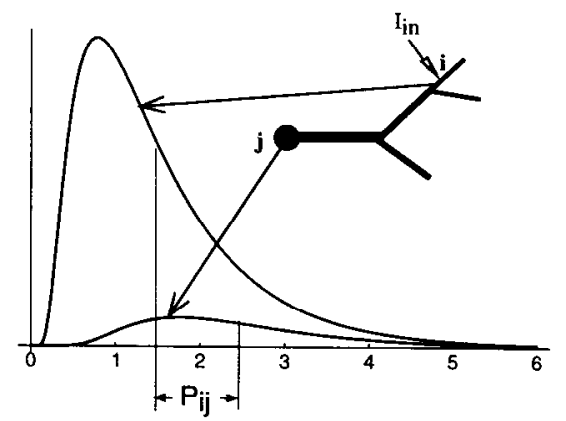

(METs) emphasize that electrotonic structure depends critically on the location of the input on the tree and on the question of interest (Zador et al., 1991; Brown et al., 1992; Zador, 1993; see also Tsai et al., 1994); indeed, for any given tree there are an infinite number of such transformations. Each MET emphasizes a different aspect of electrotonic structure, yet consideration of just a few can provide a rather complete functional view of a neuron's tree. Applying the MET to different neuron types (layer 5 and layer $2 / 3$ cortical pyramidals, hippocampal pyramidal, and cerebellar Purkinje), we gain insights into the different integrative properties of these neurons. Finally, we show that the MET can be cxtended to treat ionic signaling in dendritcs and spines.

\section{Theory}

\section{Model assumptions}

The formalism developed here assumes that the electrical potential along each branch satisfies the standard one-dimensional cable equation (Jack et al., 1983; Rall, 1989):

$$
\tau_{m} \frac{\partial V(x, t)}{\partial t}=\lambda^{2} \frac{\partial^{2} V(x, t)}{\partial x^{2}}-V(x, t)-I\left(x_{0}, t\right) R_{i n},
$$

where $V(x, t)$ is the membrane potential at time $t$ and position $x, I\left(x_{0}, t\right)$ is the injected current at some point $x_{0}$ where the input resistance is $R_{i n}, \tau_{m}=R_{m} C_{m}$ is the membrane time constant, $\lambda$ $=\sqrt{d R_{m} / 4 R_{i}}$ is the space constant, $d$ is the process diameter, and $C_{m}, R_{m}$, and $R_{i}$ are the membrane capacitance, resistivity, and axial resistance, respectively. Note that the formalism below permits the current $I\left(x_{0}, t\right)$ to be generated by transient conductance changes, for example, by a synaptic input $I\left(x_{0}, t\right)=$ $g_{\text {syn }}(t)\left[V\left(x_{0}, t\right)-E_{\text {syn }}\right]$.

\section{Definitions}

Electrotonic distance and length. Here we review the standard definitions of electrotonic distance and length (Rall, 1959; Jack et al., 1983). The electrotonic distance $X$ is the physical distance $x$ scaled by the length constant $\lambda$ :

$$
X=\frac{x}{\lambda} .
$$

Similarly, the electrotonic length of a finite cable is the total length $l$ scaled by the length constant

$$
L=\frac{l}{\lambda} \text {. }
$$

A more general expression (Rall, 1962) for $X$ between two points $i$ and $j$, applicable to cables of varying geometry or membrane properties, is

$$
X=\int_{x_{i}}^{x_{j}} \frac{d x}{\lambda(x)}
$$

Transfer impedances. Here we present the two-port formulation of electrotonic structure (Butz and Cowan, 1974; Carnevale and Johnston, 1982; Koch et al., 1982). Let $i$ and $j$ be spatial indices along a branched structure without loops. If we inject an arbitrary current $I_{i}(t)=I\left(x_{i}, t\right)$ at some location $i$, then the voltage $V_{j}(t)=V\left(x_{j}, t\right)$ at any other point $j$ in response to $I_{i}$ is

$$
V_{j}(t)-\int_{-\infty}^{\infty} I_{i}(\tau) K_{i j}(t-\tau) d \tau=I_{i}(t) * K_{i j}(t),
$$

where $*$ indicates convolution. $K_{i j}(t)=K\left(x_{i}, x_{j}, t\right)$ is the response at $j$ to a Dirac delta pulse $\delta(t)$ of current injected at $i$. $K_{i j}$ (which has units of $\Omega$ ) is the transfer impedance (also called the impulse response or Green's function) from $i$ to $j$. The convolution becomes a multiplication following the Fourier transform of Equation 5 ,

$$
\tilde{V}_{j}(f)=\tilde{I}_{i}(f) \tilde{K}_{i j}(f)
$$

where $\tilde{K}_{i j}(f)$ is the Fourier transform of $K_{i j}(t)$ (We will in general denote the Fourier Transform of a function $y(t)$ by $\tilde{y}(f)$, and the steady state by $\tilde{y}(0)$ or simply $\tilde{y})$. In the frequency domain $\tilde{K}_{i j}(f)$ is in general a complex number even though in the time domain $K_{i j}(t)$ is real.

For many purposes the quantities of primary intercst arc the steady-state values $\tilde{V}_{i}(0)$ and $\tilde{I}_{i}(0)$. For transient signals $\tilde{V}_{i}(0)$ is the integral of voltage at the point $i$, that is, the area under the voltage transient. Similarly, $\tilde{I}_{i}(0)$ is the integral of injected current at the point $i$, that is, the total injected charge. At the point of current injection the Green's function takes a special name, the input resistance, given by $R_{i n}=\tilde{K}_{i i}(0)$.

Log-attenuation. The voltage attenuation between two points $i$ and $j$ (Fig. 1A) is defined as (Koch et al., 1982)

$$
A_{i j}=\frac{\tilde{V}_{i}}{\tilde{V}_{j}}=\frac{\tilde{K}_{i i}}{\tilde{K}_{i j}}
$$

The first subscript $i$ gives the location of the current input, and the second subscript $j$ the location of the recording electrode. Taking the logarithm, we then define the log-attenuation as 


$$
L_{i j}=\log A_{i j}=\log \frac{\tilde{V}_{i}}{\tilde{V}_{j}} .
$$

Since the response is always greatest at the site of injection, $\tilde{V}_{i}$ $\geq \tilde{V}_{j}$ so that $L_{i j} \geq 0$.

The formalism developed below applies without modification to the frequency dependent attenuation, $A_{i j}(f)=\tilde{K}_{i i}(f) / \tilde{K}_{i j}(f)$, and the frequency-dependent $\log$-attenuation $L_{i j}(f)=\log \left|A_{i j}(f)\right|$. Note that in general $A_{i j}(f)$ is a complex number, but $L_{i j}(f)$ is real. For completeness we observe that the phase-lag $T_{i j}(f)=$ $\arg \left(A_{i j}(f)\right)$ has entirely analogous properties, but we will not develop them further in this article (Zador, 1993).

An expression for $L_{i j}$ analogous to Equation 4 is

$$
L_{i j}=\int_{x_{i}}^{x_{j}} \frac{d x}{\lambda_{e f f}(x)} .
$$

where the effective space constant $\lambda_{\text {eff }}=\mid \tilde{V}(f=0, x) /(d \tilde{V}(f=$ $0, x) / d x) \mid($ Agmon-Snir, 1994) is the functional generalization of the classical definition for $\lambda$.

Centroids and delays. Centroids and delays are useful measures of the effect of electrotonic structure on the timing of signals (see Agmon-Snir and Segev, 1993, for details). We define the centroid $\hat{t}_{i}$ (Fig. $1 B$ ) of a transient signal $s_{i}(t)$ at a point $i$ as

$$
\hat{t}_{i}^{s}=\frac{\int_{-\infty}^{\infty} t s_{i}(t) d t}{\int_{-\infty}^{\infty} s_{i}(t) d t}
$$

The centroid can be interpreted as the "average" or the "center of gravity" of the signal. We will use the subscript to denote the spatial location at which the centroid is computed, and the superscript to denote the signal type. Thus, $\hat{t}_{i}^{I}$ is the centroid of some transient current $I_{i}(t)$ applied at $i$, and $\hat{t}_{i}^{V}$ the centroid of the voltage response at that point. As an example, Figure $1 B$ shows the centroid $\hat{t}_{i}^{v}$ of the voltage response to a transient current input at the point of injection, and the centroid $\hat{t}_{j}^{V}$ at some other point $j$ in the tree.

We define delay as the difference between centroids. By analogy with the transfer impedance, we define the total or transfer delay as the difference between the input centroid $\hat{t}_{i}^{l}$ at $i$ and the response centroid $\hat{t}_{j}^{V}$ at $j$,

$$
D_{i j}=\hat{t}_{i}^{V}-\hat{t}_{i}^{I}
$$

In a passive neuron $D_{i j}$ is always positive. The local or input delay is defined by analogy with the transfer impedance as $D_{i i}$, that is, the transfer delay at the point $i$. The propagation delay $P_{i j}$ is defined as the difference between voltage centroids at two points $i$ and $j$,

$$
\begin{aligned}
P_{i j} & =\hat{t}_{j}^{V}-\hat{t}_{i}^{V} \\
& =D_{i j}-D_{i i}
\end{aligned}
$$

It is a measure of the propagation rate of the voltage centroid along the tree.

By analogy with Equations 4 and 9 we can express the propagation delay in integral form,

$$
P_{i j}=\int_{x_{i}}^{x_{j}} \frac{d x}{\theta(x)},
$$

where $\theta(x)=\lambda_{e f f}(x) / \tau_{e f f}(x)$ is the propagation velocity at $x$. The effective time constant $\tau_{\text {eff }}$ is the functional generalization of the classical $\tau_{m}$ (Agmon-Snir, 1994).

The total delay and the propagation delay between two points in a passive structure are independent of the shape of the transient signal (Property 9 below). Also, $P_{i j}$ has structural invariance property (Property 4 below) and additivity property (Property 5 below). Without these properties, the MET could not be applied to delay analysis. If the peak time, for example, were used in the definitions of the delay, these properties would not hold. In the Results section below, we show that these delays, defined with respect to the centroid, have an interesting physiological interpretation. A fuller discussion of the relationship between the centroid and classical measures of dendritic transients (e.g., time-to-peak, half-width) can be found in AgmonSnir and Segev (1993).

\section{Properties of transfer impedance, log-attenuation, and delay}

Here we catalog briefly some important properties of the quantities defined above that will be useful in interpreting the morphoelectrotonic transform. Detailed references and proofs may be found in (Torre and Poggio, 1978; Koch et al., 1982; AgmonSnir and Segev, 1993; Zador, 1993).

The first two properties describe the symmetries and asymmetries of these measures within the tree.

Property 1 (asymmetry). The propagation delay and the logattenuation are not in general symmetric:

$$
\begin{aligned}
& L_{i j} \neq L_{j i} \\
& P_{i j} \neq P_{j i} .
\end{aligned}
$$

These can be verified by applying the definitions of $L_{i j}$ (Eq. 7) and $P_{i j}$ (Eq. 12). Inequality holds whenever (for $L_{i j}$ ) $\tilde{K}_{i i} \neq \tilde{K}_{j j}$ or (for $P_{i j}$ ) $D_{i i} \neq D_{i j}$. Infinite cylinders are an exception, since in this case $\tilde{K}_{i i}=\tilde{K}_{j j}$ and $D_{i i}=D_{i j}$.

Property 2 (symmetry). The transfer impedance $\tilde{K}_{i j}$ and the transfer delay $D_{i j}$ are symmetric:

$$
\begin{aligned}
& \tilde{K}_{i j}=\tilde{K}_{j i} \\
& D_{i j}=D_{j i} .
\end{aligned}
$$

The next three properties relate these quantitics at onc position along the cable to other positions.

Property 3 (on-path impedances). If $j$ is a point on the path between $i$ and $k$, then the transfer impedance from $i$ to $k$ can be computed directly from the transfer impedances from $i$ to $j$ and $j$ to $k$ :

$$
\tilde{K}_{i k}=\frac{\tilde{K}_{i j} \tilde{K}_{j k}}{\tilde{K}_{j j}}
$$

This is easily shown by algebraic manipulation of Equation 6 .

Property 4 (structural invariance). Let $j$ be a point on the path between $i$ and $k$. Then $P_{i j}$ and $L_{i j}$-the measures directed toward $k$-depend on the electrotonic properties at $k$, but $P_{j i}$ and $L_{j l}$ the measures directed away from $k$-do not. This property says that the $L_{i j}$ and $P_{i j}$ depend on the electrotonic structure "in front" of $i$ but not "behind" $i$.

Property 5 (additivity). If a point $j$ is on-path from $i$ to $k$, then the total log-attenuation and the total propagation delay are just the sum of the respective partial measures. That is, the logattenuation and the delay are additive for $j$ between $i$ and $k$, 


$$
\begin{aligned}
& L_{i k}=L_{i j}+L_{j k} \\
& P_{i k}=P_{i j}+P_{j k} .
\end{aligned}
$$

These expressions can be verified from the definitions (Eq. 16 and Eqs. 11 and 12). In this respect, these measures behave as a Euclidean distance metric between collinear points. However, because of asymmetry (Property 1), neither is a true distance metric in the mathematical sense (except in the case of an infinite cylinder), since the "distance" from $i$ to $j$ is not equal to the "distance" from $j$ to $i$.

The next two properties describe dependencies on fiber size and input frequency.

Property 6 (high-pass filtering). Since the transfer impedance acts as a low-pass filter, higher frequencies are more attenuated than lower:

$$
\left|L_{i j}(f)\right| \geq\left|L_{i j}\left(f^{\prime}\right)\right| \text { for } f>f^{\prime} .
$$

That is, the log-attenuation is a monotonically increasing function of frequency.

Property 7 (dendritic size effects). If the input impedance is higher at a point $i$ (e.g., in the dendrites) than at a point $j$ (e.g., at the soma), $\tilde{K}_{j j}<\tilde{K}_{i,}$, then

$$
L_{i j}>L_{j i} \text {. }
$$

Similarly, if the local delay is shorter at a point $i$ (e.g., in the dendrites) than at a point $j$ (e.g., at the soma), $D_{i i}<D_{j j}$, then

$$
P_{i j}>P_{j i}
$$

These properties imply that if the typical size relations between the soma and dendrites pertain, then signal transfer is more effective away from than toward the soma (Rall and Rinzel, 1973; Nitzan et al., 1990).

We conclude with two properties that emphasize that applicability of these measures to arbitrary transient signals.

Property 8 (log-attenuation of transient signals). The log-attenuation from $i$ to $j$ of total charge for any transient signal is $L_{j i}$,

$$
L_{j i}=\log \frac{\int_{0}^{\infty} I_{i}(t) d t}{\int_{0}^{\infty} I_{j}(t) d t} .
$$

Similarly, the log-attenuation for $i$ to $j$ of the area under the voltage transient is $L_{i j}$,

$$
L_{i j}=\log \frac{\int_{0}^{\infty} V_{i}(t) d t}{\int_{0}^{\infty} V_{j}(t) d t} .
$$

The numerator on the right side of Equation 21 is the total charge injected by the transient signal at $i$, and the denominator is the total charge received at $j$ when the potential at $j$ is clamped. The logarithm of their ratio, which is independent of the time course of the injected signal, is just equal to the voltage transfer in the opposite direction (Koch et al., 1982). From the Properties 6 and 7 , it follows that the charge attenuation will typically be the best somatic measure (i.e., the minimum attenuation) of a synaptic event (Rinzel and Rall, 1974).

Property 9 (shape invariance). The delay $D_{i j}$ and $P_{i j}$ of a tran-

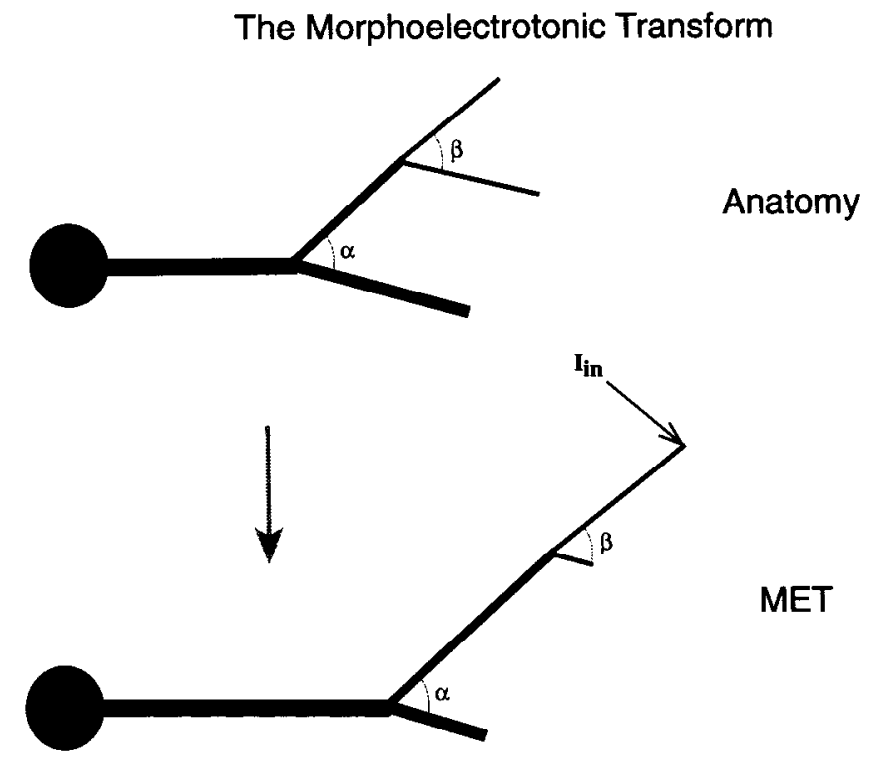

Figure 2. The morphoelectrotonic transform (MET) maps from anatomical to functional space. A neuron can be represented as a collection of line segments of known lengths and diameters, connected at fixed angles. In a MET, the angles and diameters are retained, but the anatomical lengths are replaced by some functional lengths. Typically the functional length depends on the site of current injection, as indicated by $I_{\text {in }}$.

sient signal are independent of the shape of the signal and depend only on the properties of the dendritic tree. This property is proved in Agmon-Snir (1994).

\section{Morphoelectrotonic transform}

In the last several years it has become possible to obtain very detailed three-dimensional reconstructions of the branching structure of a dendritic tree (Fleshman et al., 1988; Douglas et al., 1991; Rapp et al., 1994). In such a reconstruction, neuronal morphology can be described as a set of short segments, with some measured length and diameter, which when connected and placed in the proper spatial orientation produce the complete dendritic tree. If we make assumptions about the membrane and cytoplasmic parameters $R_{m}, C_{m}$, and $R_{i}$, the reconstructed neuron provides a full description of its passive properties (Rall et al., 1992).

Because of the tremendous wealth of data made available by a reconstructed neuron, it is often difficult to infer electrotonic function from morphological structure. We have therefore developed a method that exploits the additivity (see Property 5) of the log-attenuation and the propagation delay as the basis for a graphical transformation, the morpho-electrotonic transform or $M E T$. The MET permits direct visualization of functional implications of electrotonic structure. In a MET, the anatomical length of each of the vectors comprising the dendritic tree is replaced by one of the additive measures, either $P_{i j}$ or $L_{i j}$, while the diameter and orientation of the segment are preserved (see Fig. 2). In this way different aspects of electrotonic structure can be instantly appreciated, and need no longer be inferred indirectly from the morphology.

In this article we will consider two primary MET subtypes. The attenogram shows signal attenuation $L_{i j}$, while the delayogram shows signal delay $P_{i j}$. In the attenogram each unit length represents $e$-fold voltage attenuation; in the delayogram, each 
unit length represents a fixed propagation delay (in msec). Since these measures are directional, each subtype is either centrifugal, showing signal transfer away from some reference point, or centripetal, showing signal transfer toward some point. Complete specification of the MET therefore requires at least three parameters: the reference point, the direction, and the subtype. For example, a centrifugal somatic attenogram shows log-attenuation of voltage away from the soma. Note that in some cases it may be necessary to specify additional parameters, such as the frequency of the sinusoidal current injected at the reference point.

\section{Implementation of the MET}

Morphological measurements of reconstructed neurons were obtained from several different laboratories in the form of computer files. From these files, generation of a MET required two steps: a calculation step to compute the electrotonic properties such as input and transfer impedances, and rendering step to display the result graphically.

For preliminary work (Zador, 1993) on the attenogram, a matrix algorithm was developed to calculate only the requisite elements of the impedance matrix $K$ (Zador and Pearlmutter, 1993). The algorithm scales linearly with the spatial discretization $n(O(n)$ in runtime and memory) and has an error proportional to $O\left(\Delta x^{2}\right)$. However, in this article all attenograms were produced using a now standard recursive algorithm developed by Rall (1959) (see also Koch and Poggio, 1985) that scales linearly with the number of neuronal branches. For the delayogram an analogous recursive algorithm was developed in (Agmon-Snir, 1994). The implementations were verified on branching structures for which the analytic solutions were known, and results agreed to within machine error. Unless otherwise specified the following default membrane parameters were used: $R_{m}$ $=20 \mathrm{k} \Omega \mathrm{cm}^{2}, R_{i}=100 \Omega \mathrm{cm}$, and $C_{m}=1 \mu \mathrm{F} / \mathrm{cm}^{2}$.

All calculations and renderings were done in MATHEMATICA (Wolfram, 1992). Code is available upon request from the authors. Comparable tools are being developed for NEURON (Hines, 1989).

\section{Results}

\section{Analytical results}

Infinite cylinder. In an infinite cylinder, the log-attenuation $L_{i j}$ is identical to the electrotonic distance $X$ between $i$ and $j$. This observation provides an intuitive basis for interpreting $L_{i j}$. The proof is straightforward and helps makes the relationships clear. We begin with the standard expression for the steady-state spread of voltage in an infinite cylinder (Rall, 1989),

$$
\bar{V}_{j}=\tilde{V}_{i} e^{-x}
$$

where $\tilde{V}_{i}$ is the steady-state voltage at the point of current injection, $\tilde{V}_{j}$ is the steady-state voltage at some point $j$, and $X$ is the electrotonic distance between $i$ and $j$. Rearranging and substituting the definition of the voltage attenuation, we have

$$
A_{i j}=\frac{\tilde{V}_{i}}{\tilde{V}_{j}}=e^{x}
$$

Finally, by taking the logarithm of both sides and substituting the definition of the log-attenuation, we obtain the equivalence

$$
L_{i j}=X \text {. }
$$

This equation provides an intuitive basis for understanding the attenogram: for the case of an infinite cylinder, the log-attenuation is just the electrotonic distance. The attenogram rescales the dendritic tree so that each unit length behaves like a segment of an infinite cylinder, across which there is an $e$-fold attenuation. Similarly, in the delayogram the tree is rescaled so that there is just a $X \tau_{m} / 2$ (Agmon-Snir and Segev, 1993) delay across each unit distance.

Finite cylinder. Consideration of $L_{i j}$ and $P_{i j}$ in a short terminal branch of length $L$ attached to a thicker branch provides a stepping stone to understanding their properties in complex dendritic trees (Fig. 3A). Transfer in two directions must be considered. Analysis of current injection at the branchpoint - out toward the tip-helps explain the case of centrifugal transfer, while injection at the distal tip-in toward the branchpoint and the somacorresponds to centripetal transfer. The case of an infinite cylinder is included for reference.

Figure $3 B$ shows the dependence of $L_{i j}$ on $L$. For the infinite cylinder the plot is just a straight line with slope of unity, since as discussed above $L_{i j}=L$ across any segment of length $l$. Injection into the branchpoint yields the lower curve, always less than the reference curve. This curve shows that attenuation across a terminal fiber of length $L$ is less than across a distance $L$ in an infinite cylinder. For example, for a terminal segment with $L=0.5, L_{i j}=0.12$, so that in a MET the segment will appear only about a quarter of its size as predicted from the classical measure of electrotonic length. By contrast, injection at the distal tip of this same branch yields the upper curve which is always greater than the reference case. For the same $L=0.5$ fiber, the log-attenuation is now $L_{i j}=1.8$, so that in a MET the fiber appears longer. Figure $3 C$ illustrates comparable effects of asymmetric signal transfer on $P_{i j}$.

In Figure $3 D$, the ratio $L_{i j} / P_{i j}$ is shown for both directions and the reference infinite cylinder. For the infinite cylinder the ratio is just $2 / \tau_{m}$, since in this case $P_{i j}=L \tau_{m} / 2$ and $L_{i j}=L$. For transfer out across short terminal segments (lower curve) the ratio is $1 / \tau_{m}$, and climbs with increasing $L$ only very gently to its limiting value of $2 / \tau_{m}$. If the terminal branches of a neuron are short $(L<1)$, then the ratio of the two measures is almost constant for transfer out. By contrast, the ratio in the case of transfer in is highly dependent on segment length, and (data not shown) on the relative diameters of the parent branches. Thus, for this latter case the attenogram can differ markedly from the delayogram.

\section{Functional views of a single pyramidal cell}

Voltage transfer from the soma. Figure 4 (left) shows an anatomical reconstruction of a layer 5 pyramidal cell from cat visual cortex (Douglas et al., 1991). It consists of a relatively short bushy basal tree, with fine processes terminating about $200 \mu \mathrm{m}$ from the soma, and a bifurcating apical tree with fine processes terminating about $1000 \mu \mathrm{m}$ from the soma. The diameters of the finest processes are near the limit of light microscopy, tapering down to about $0.2 \mu \mathrm{m}$.

In Figure 4 (middle), the physical length $x$ of each segment has been replaced by its electrotonic length, $X=x / \lambda$. We call this the "classical attenogram" because it replaces the anatomical distance with the classical measure $\lambda$. It does not provide a direct measure of the efficacy of signal transfer, since only in an infinite cylinder does the attenuation of voltage bear a simple direct relationship to the electrotonic length. This is not, therefore, a functional transformation. Since $\lambda$ depends only on the fiber diameter-thinner processes have shorter space constants so they are electrotonically longer-this transformation is merely a way of representing the relative fiber diameters. Therefore, the fine processes in the basal and the distal apical trees are 
Figure 3. $L_{i j}$ and $P_{i j}$ can be understood in terms of a short segment attached to a larger branch. In the simplified model considered here $(A)$, current was injected into either the distal end (in) or proximal branchpoint (out) of a short segment of length $L$ attached to a larger branch which acts as a current sink. For comparison, a third case of an infinite eylinder $(L \rightarrow$ $\infty)$ is also included. In mathematical terms, these cases can be considered boundary conditions parameterized by $\rho_{\infty}$ and $\epsilon$ (see Eq. 25 in Rall, 1989, and Eq. 15 in Agmon-Snir and Segev, 1993). Injection at the branchpoint corresponds to the sealed-end case $\left(\rho_{\infty} \rightarrow\right.$ $\infty$ and $\epsilon=0.5$ ). Injection at the distal tip looking toward the soma corresponds to the leaky-end case $\left(\rho_{\infty}=0.1\right.$ and $\epsilon=0.5$ ). For the reference case we used $\rho_{x}=1$ and $\epsilon=0.5$. B shows the dependence of $L_{i j}$ on $L$ for injection at the branchpoint (lower curve), at the distal tip (upper curve), and for $L \rightarrow \infty$ (dashed line). Similarly, $C$ shows the dependence of $P_{i j}$ on $L$ for injection at the branchpoint (lower curve), at the distal tip (upper curve), and for $L \rightarrow \infty$ (dashed line). In $D$, the ratio of $L_{i j} / P_{i j}$ is plotted for injection at the junction (lower curve), at the distal end (upper curve), and into an infinite cylinder (dashed line).
A

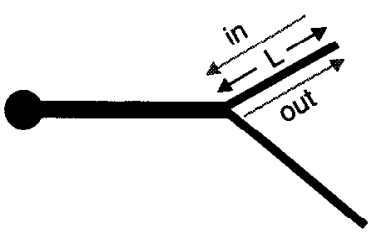

C

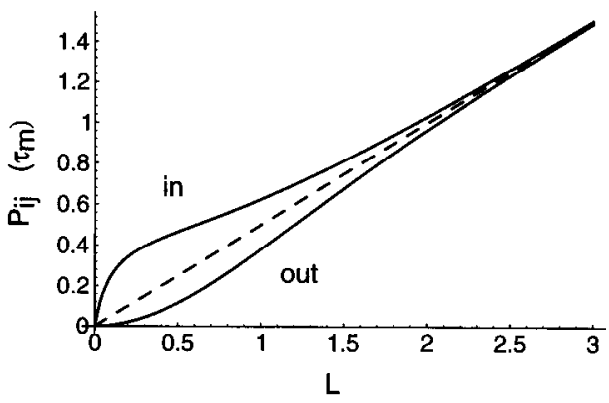

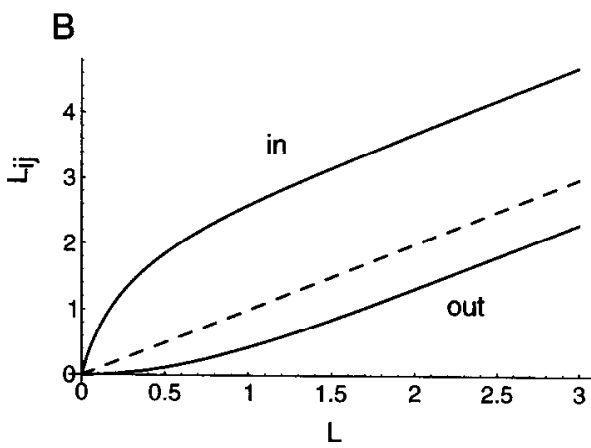

D

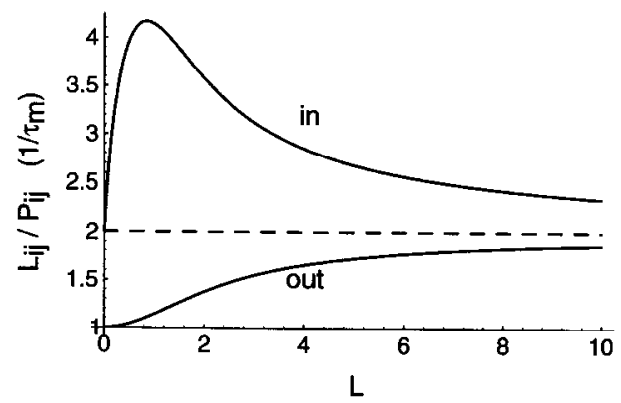

relatively elongated, while the thick apical trunk is relatively compressed. This transformation is a useful bridge between the real morphology and the functional transformations to follow.

Figure 4 (right) shows the centrifugal attenogram $\left(L_{s d}\right.$ from the soma to the dendrites). For each segment, the anatomical length has been replaced by the corresponding log-attenuation of voltage away from the soma. This is a truly functional transformation, in that the voltage transfer can be read directly from the figure. By the asymmetry property (Property 1), the attenogram is directional; this one illustrates only the transfer of potential away from the soma. The scale, in units of $\lambda_{e f f}$ is shown in the figure (see calibration bar): for each $0.1 \lambda_{\text {eff }}$ unit length, the voltage decreases by a factor of $e^{-0.1}=0.9$ relative to the soma. The tips of even the most distal basal processes are close; the attenuation is no more than about $e^{-0.05}=0.95$. By contrast, the tips of the most distal apical processes are about 0.6 units from the soma, so that the voltage attenuation from the soma to these distal dendrites is about a factor of two $\left(e^{-0.6}=0.55\right)$. For example, if a hyperpolarizing stimulus at the soma causes a -20 $\mathrm{mV}$ drop there from $-70 \mathrm{mV}$ to $-90 \mathrm{mV}$, the distal tips can be expected to drop only by $-10 \mathrm{mV}$, to $-80 \mathrm{mV}$. Nevertheless, the overall scale suggests that, with respect to this measure, the neuron is electrotonically compact.

Figure 4 (right) reveals that transfer of somatic voltage is very effective to the basal tree, but less so to the apical tree, particularly along the fine distal apical branches. This contrast is not immediately apparent from the original morphology (Fig. 4, left), in which the basal tree is relatively longer, and the distal

Figure 4. The centrifugal attenogram of a cortical pyramidal neuron differs from its classical transformation. A three-dimensional reconstruction of a pyramidal cell from layer 5 of cat visual cortex (left; from Douglas et al., 1991) was transformed according to two different measures. Color here is a visual aid that helps identify corresponding segments in the two transforms and the original anatomy. In the classical transformation (middle), the anatomical length of each process was replaced by its electrotonic length $L=l / \lambda$, which depends only on the fiber geometry. This shows how the neuron would behave in the absence of boundary effects, that is, if every process were an infinite cylinder. Thinner processes in the basal tree and the distal apical tree are relatively elongated. This is not a functional transformation. By contrast, the centrifugal (out away from the soma) attenogram (right) is a functional transformation that illustrates the $\log$-attenuation $L_{s d}=\log \left(\tilde{V}_{s} / \tilde{V}_{d}\right)$ of voltage from the soma at $s$ (arrow) to every other point $d$. The somatic view (input to the soma, looking out) is indicated by the schematic current electrode $\left(I_{i n}\right)$. The scale of the classical transformation is in units of $\lambda$ while for the attenogram it is in units of $\lambda_{\text {eff }}$ (see text). Since the two transformations are drawn to the same scale, the sinaller size of the functional attenogram reflects greater electrotonic compactness due to boundary conditions. The architecture of the two transforms are also markedly different, particularly with regard to the relative sizes of the apical and basal trees. Default parameters were used: $R_{m}=20 \mathrm{k} \Omega \mathrm{cm}^{2}, R_{i}=100 \Omega \mathrm{cm}$, and $C_{m}=1 \mu \mathrm{F} / \mathrm{cm}^{2}$.

Figure 5. Centripetal transforms differ in form and scale from their centrifugal counterparts. On the left, the centripetal somatic attenogram (in toward the soma) shows $L_{d s}$ from every point to the soma. The somatic view is indicated by a schematic recording electrode (arrow) at the soma for the hypothetical experiment of injecting current independently at each of the dendritic tips. The 10-fold larger scale compared with the centrifugal transform (included to scale from Fig. 4, right; see inset) indicates that transfer of voltage toward the soma is much less effective than away from the soma. The form also gives greater emphasis to the basal tree relative to the apical tree. Similar changes can be observed in the centripetal somatic delayogram (right), shown with its centrifugal counterpart to scale (inset; see Fig. 6). As above, color is used as a visual aid to help identify corresponding segments in the two transforms. Default parameters were used: $R_{m}=20 \mathrm{k} \Omega \mathrm{cm}^{2}, R_{i}=100 \Omega \mathrm{cm}$, and $C_{m}=1 \mu \mathrm{F} / \mathrm{cm}^{2}$. 

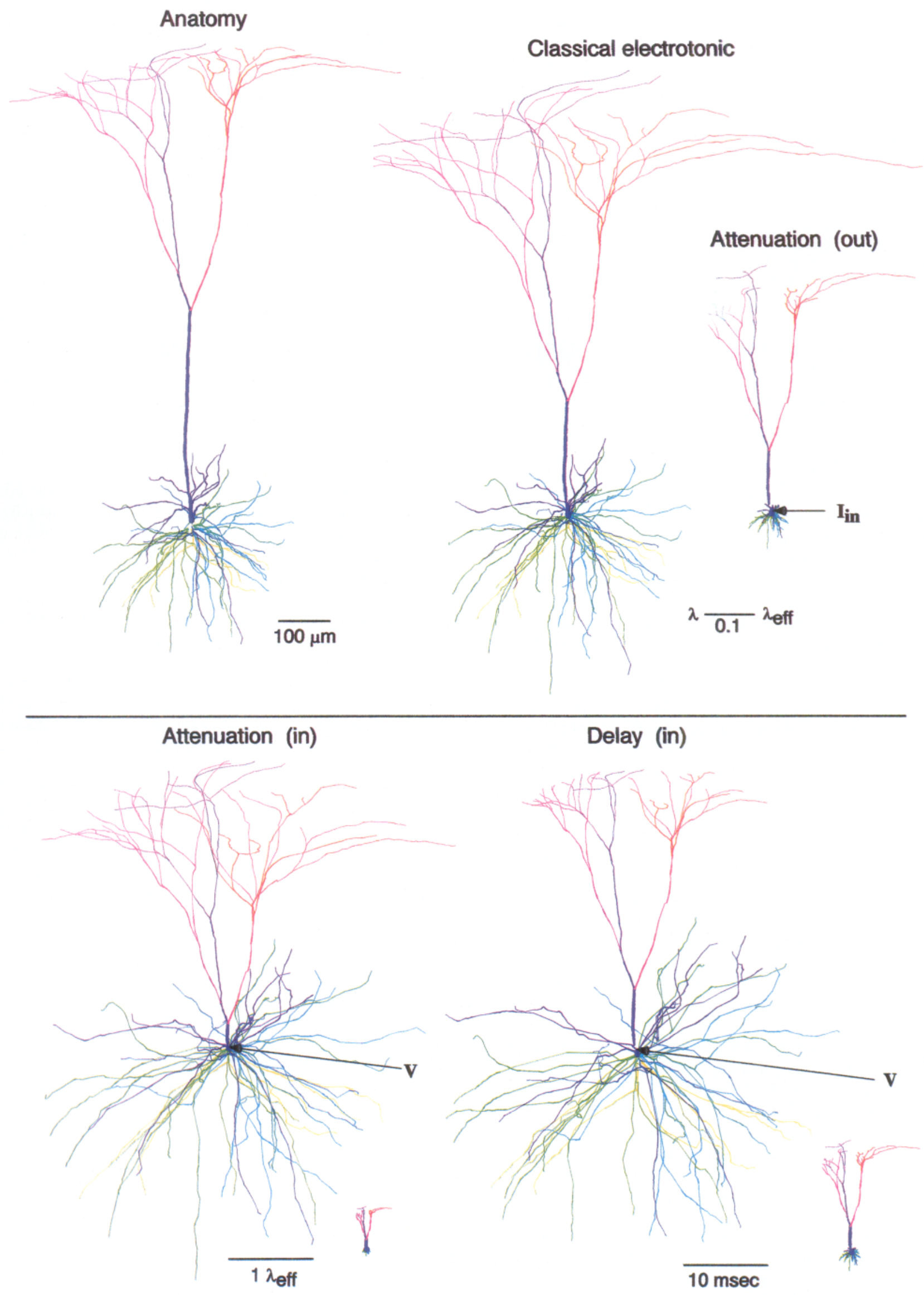
Figure 6. Centrifugal delay in a cortical neuron differs from its classical transformation. In the classical transformation (left), anatomical length was replaced by the delay $\left(X \tau_{m} / 2\right)$ assuming each segment behaves as an infinite cylinder. This view is identical to Figure 4 (middle) times a scale factor $\left(\tau_{m} / 2\right)$. In the centrifugal delayogram $($ right $)$, the length was replaced by the propagation delay $P_{\text {sd }}$ out away from the soma across each segment. Note that the form of the delayogram is almost indistinguishable from that of the attenogram, with the same effects on the apical and basal trees. Default parameters were used: $R_{m}=20 \mathrm{k} \Omega \mathrm{cm}^{2}$, $R_{i}=100 \Omega \mathrm{cm}$, and $C_{m}=1 \mu \mathrm{F} / \mathrm{cm}^{2}$.
Classical delay

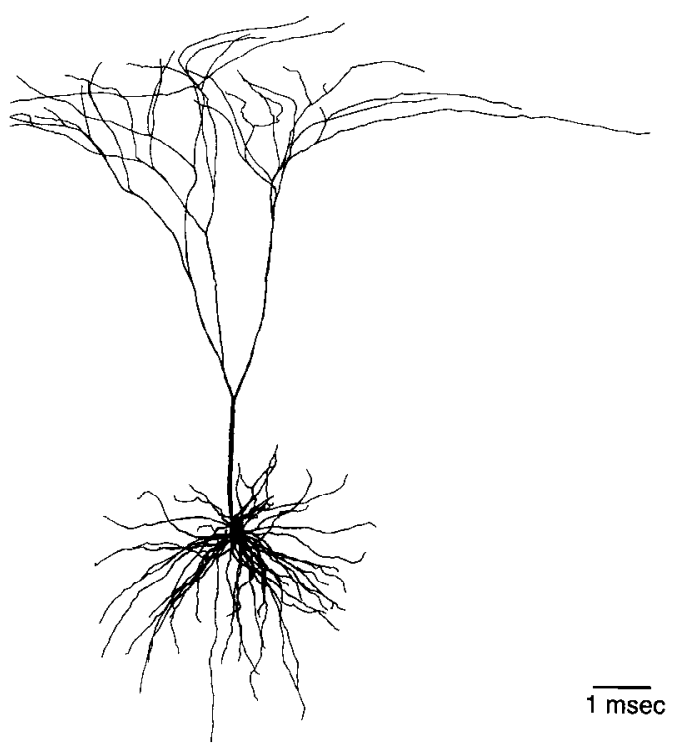

Delay (out)

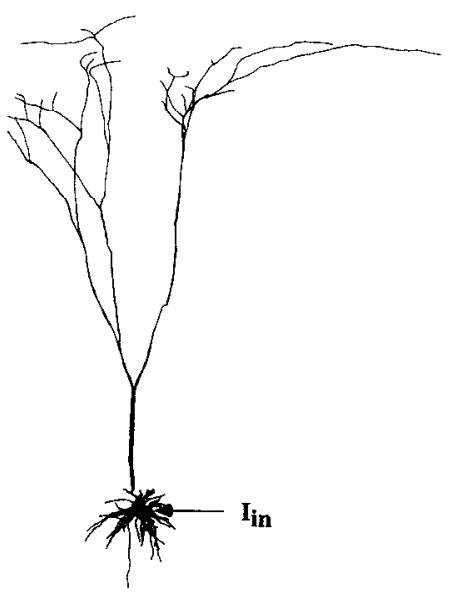

processes are of different proportions. What accounts for these effects? The interplay of two factors give rise to the final picture. First, distal processes tend to be finer, which increases their effective length; only this effect is captured in the classical attenogram (4, middle). Second, boundary effects on short terminal tend to decrease their effective length (see Fig. 3). Figure 4 (right) shows that in the basal tree the boundary effects dominate, resulting in a marked contraction with respect to the classical attenogram, while in the apical tree diameter plays a greater role because the processes are longer and thinner.

This MET also has another interesting functional interpretation: it illustrates the cost in terms of somatic efficacy of moving an input from the soma to any dendritic site. Formally, the responses at the soma due to the inputs at the soma and the dendrite is $\tilde{V}_{s}=\tilde{K}_{s s} \tilde{I}_{s}$. When the same current is applied to the dendrites $\left(\tilde{I}_{s}=\tilde{I}_{d}\right)$ the resultant voltage at the soma is $\tilde{V}_{s}^{\prime}=\tilde{K}_{d s} \tilde{I}_{d}$ $=\tilde{K}_{s d} \tilde{I}_{s}$. Then their ratio is just $\tilde{V}_{s} / \tilde{V}_{s}^{\prime}=\tilde{K}_{s s} / \tilde{K}_{s d}=A_{s d}$. For example, suppose that at the distal tip there is a twofold attenuation of voltage. Then if some current input $\tilde{I}_{0}$ applied at the soma produces a somatic depolarization of $1 \mathrm{mV}$, then the same current applied at the distal tip produces only half the somatic depolarization. Thus this attenogram shows how much less effective an input is in the dendrites than it would have been if placed directly at the soma. In the case of Figure 4, the cost of placing the input on the basal tip is about $5 \%$ and on the apical tip about $45 \%$.

Propagation delay from the soma. Figure 6 (right) shows the propagation delay $P_{s d}$ for a signal applied at the soma. The most straightforward interpretation of this delayogram is similar to that of the corresponding attenogram, except that here the scale has units of time (calibration is $1 \mathrm{msec}$ ) while in the attenogram the scale is unitless. The figure shows, for example, that the centroid of a somatic signal takes about $20 \mathrm{msec}$ to propagate to the most distal apical tips, but only about $1 \mathrm{msec}$ to the basal tips.

This delayogram also has two other interesting interpretations. First, as in the case of the centrifugal attenogram, it represents the cost in terms of delay of moving a stimulus from the soma to any site in the dendrites. For example, if the input delay $D_{s s}$ at the soma is $14 \mathrm{msec}$, and the transfer delay $D_{d s}$ from some point $d$ to the soma is $22 \mathrm{msec}$, then by Equation 12 the additional delay $(8 \mathrm{mscc})$ that results from moving the input from the soma to point $d$ is precisely the propagation delay from the soma, $P_{s d}$ (Agmon-Snir and Segev, 1993). Second, this delayogram also provides an approximation to the propagation delay of the signal peak, in the opposite direction, from the dendrite to the soma. This interpretation can be very useful, since the peak is so much easier to visualize than the centroid (AgmonSnir and Segev, 1993).

It is striking how similar the centrifugal delayogram and attenograms are (cf. Figs. 4, right, and 6, right). Because of this similarity, the analysis of the attenogram-where the trade-off between processes diameter and boundary effects was considered - can be applied directly to the delayogram. But why are the two transformations so similar? The explanation lies in the ratio $L_{i j} / P_{i j}$ between the delay and the log-attenuation across each segment. To the extent that this ratio is constant for all segments, the relative sizes of the segments are preserved and the transformed trees are similar up to a scale factor. As shown in Figure $3 D$ (lower curve), in a terminal segment this ratio starts at $1 / \tau_{m}$ and increases gradually with electrotonic length to $2 / \tau_{m}$. For small $L$ (e.g., $<1$ ) $P_{i j} / L_{i j}$ is close to unity. Thus, most processes appear identical in the two views. By analogy with the classical attenogram we include for comparison a "classical delayogram" (Fig. 6, left), in which each segment is treated as part of an infinite cylinder. This transformation was obtained by rescaling the classical attenogram by $\tau_{m} / 2$.

Signaling toward the soma. We have emphasized that voltage transfer is asymmetric (Property 1), and that in general, the voltage transfer out from the soma is more effective than the voltage transfer in to the soma (Property 7). These principles are illustrated dranatically in Figure 5 (left). The overall structure is markedly different from its complement (Fig. 4, right), and the scale has increased an order of magnitude (see inset). The previous centrifugal attenogram showed voltage transfer toward the tips, and therefore was dominated by the sealed-end boundary effects shown in Figure $3 B$. Here, the transfer away from tips is dominated by the electrical load that the rest of the tree imposes on each process. Since the basal processes are electrically close to the soma, they experience a large electrical sink due to the 
soma and other basal dendrites and so tend to become relatively lengthened. Thus, the apical stalk has become very short, and the basal tree has blossomed from a tuft of almost insignificant proportions to rival the apical tree.

The net result is very surprising if we base our intuitions on either the morphology or the classical attenogram (Fig. 4), which would seem to suggest that apical inputs should be far less effective than the basal inputs since they are so much farther away. In this functional transform, inputs to the basal and apical trees have comparable effects at the soma. Why are the differences between the basal and apical trees so much less marked in this attenogram than in the original morphology? How can we reconcile this functional view with the original morphology? The answer rests in part with the scale of the figure. Careful examination reveals that the most distal apical tips still remain farther than the most distal basal tips, by a factor of $e^{1} \approx 3$ (see scale bar) or more, so that these most distal apical tips are indeed less effective. Nevertheless, there is substantial overlap between the efficacies of the two trees. This attenogram emphasizes how wary we must be of drawing inferences directly from the morphology.

The delayogram (Fig. 5, right) shows the propagation delay toward the soma. The main result is similar to the corresponding attenogram (Fig. 5, left): the differences between the basal and apical trees have been diminished, so that the most distal tips in either tree have propagation delays of about $20 \mathrm{msec}$. But in contrast to the centrifugal transforms, where the attenogram was almost indistinguishable from the delayogram, in the present case the two transforms are appreciably different. The explanation rests once again in the ratio $L_{i j} / P_{i j}$ : in the centripetal transform this ratio is dominated by the electrical load. As shown in Figure $3 D$ (upper curve), for centripetal transfer this ratio is steeply dependent on $L$ [and on the load itself (not shown)]. Since the ratio is not constant there is no reason to expect that the two transforms should be similar in their details.

Electrotonic structure depends on the viewpoint. The previous two METs were somatocentric in that the transformation was performed with respect to a stimulus or observation site at the soma. This initial choice of the soma was convenient but arbitrary, since within the two-port framework the soma has no privileged status. Each site in the tree has a unique perspective on the electrical activity in the neuron. This is demonstrated in Figure 7 (left), which shows the attenogram from a tip in the basal dendritic tree. This view appears at first radically different from the somatic centrifugal attenogram: a long tail now seems to extend from the soma, and the rest of the neuron appears to have shrunk. Similarly, the centrifugal attenogram from a point in the apical tree (Fig. 7, right) shows a long apical tail several times the length of the rest of the neuron. In both cases the neuron to which the tail is connected appears rather like the centrifugal somatic attenogram, but smaller. These tails emphasize how poor voltage transfer is to the soma, and to points beyond the soma.

The transform from each of the 100 or so tips yields a different attenogram. Must we therefore generate separate attenograms from each tip-or worse, from every location in the neuron-in order to fully appreciate the neuron's electrotonic structure? We need not, for in fact the attenogram from any tip can be deconstructed into two components: the path from the tip to the soma, and the path from the soma out to every other tip. But we already know how long these paths arc, for the former is just the length of centripetal dendrosomatic segment

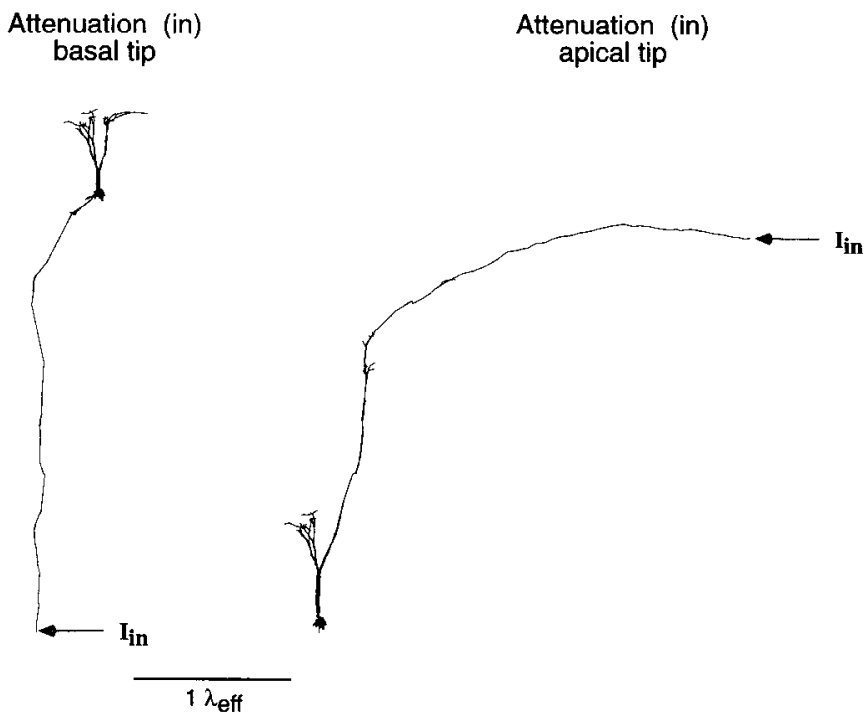

Figure 7. Voltage transfer depends on the viewpoint. Centrifugal $L_{i j}$ in to the soma is shown from a point in the basal tree (left) and the apical tree (right). The site of current injection is indicated by the schematic electrode $\left(I_{i n}\right)$. In both cases, the attenuation from the tip to the soma dominates the transform. The attenogram from any point can be constructed from pieces of the (centrifugal/centripetal) attenogram pair from one point (see text). The dendrosomatic segment of each figure is taken from the centripetal attenogram, while the rest of the figure is just the centrifugal attenogram redrawn to the appropriate scale. Default parameters were used: $R_{m}=20 \mathrm{k} \Omega \mathrm{cm}^{2}, R_{i}=100 \Omega \mathrm{cm}$, and $C_{m}=1$ $\mu \mathrm{F} / \mathrm{cm}^{2}$

in Figure 5 (left), while the latter is the rest of the centrifugal tree shown in Figure 4 (right). As noted above, the scales of the two figures differ markedly. Thus, on the scale appropriate for the single inserted dendrosomatic segment, the attenuation across the rest of the neuron is small; the inserted segment dominates the attenuation to all other locations. The argument for the delayograms are completely analogous. The key point is that a centrifugal/centripetal pair of METs from any point completely and conveniently specifies all the possible METs from every other point.

Membranes act as low-pass filters. Propagation of sinusoidal inputs can also be analyzed. From Fourier's theorem we know that any physically relevant signal can be completely decomposed into a sum of sinusoids. We can therefore consider the effect of the tree on each sinusoid independently by generating its characteristic attenogram.

Figure 8 shows somatic centrifugal attenogram in response to a sinusoidal current $(f=100 \mathrm{~Hz}$ and $f=500 \mathrm{~Hz}$ ) injected at the soma. Here attenuation refers to the peak-to-peak height of the sinusoidal, which is maximal at the point of injection (the soma). The overall form differs little from the analogous steadystate attenogram, but the scale has increased dramatically. This is a direct consequence of the filtering characteristics of dendritic membrane: high-frequencies are more attenuated than low. As the space constant decreases with frequency, boundary effects become less important and the form of the MET approaches the classical transform, but with a much longer space constant. In the limit as $f \rightarrow \infty$, boundary effects vanish and the centrifugal and centripetal transforms converge.

\section{Comparing different neurons}

The morphoelectrotonic transform also provides a rapid and direct means for comparing different classes of neurons. We com- 
Figure 8. Attenograms in the frequency domain reflect low-pass filtering by the membrane. Sinusoidal currents injected at the soma were used to construct centrifugal attenograms. The attenuation is defined with respect to the peak-to-peak height of the sinusoidal response. Because of low-pass membrane filtering effects, the scale of the attenuation is much greater for $f=$ $500 \mathrm{~Hz}$ (right) than for $f=100 \mathrm{~Hz}$ (left). As $f$ increases, the frequency dependent space constant decreases and boundary effects become less important. In the limit $f \rightarrow \infty$, the transform converges to the classical transform in its shape.

pared the layer 5 cortical cell discussed above with neurons from three widely disparate classes-layer $2 / 3$ cortical pyramidal, cerebellar Purkinje, hippocampal CA1 pyramidal-to provide a broad overview (Fig. 9). Our goal here is not an exhaustive analysis of the differences between these classes, so we point out only the highlights based a single view, the somatic centrifugal attenogram.

Figure 9 shows the four classes drawn to anatomical scale (left column), morphoelectrotonic scale (middle column), and normalized for comparison (right column). The differences in scale between the four classes is striking. The Purkinje cell has been reduced almost to a point while the apical stalk of hippocampal pyramidal cell has sprouted. The cortical cells are intermediate. The scaled view is a convenient way of assessing the relative electrotonic compactness of different neurons.

The normalized view (right column) emphasizes the variable degree to which neuronal architecture is distorted by the MET. In some (e.g., layer $2 / 3$ pyramidal) the morphology does not differ dramatically from the attenogram. In others (e.g., Purkinje, CA1 pyramidal) the difference is striking. In these latter attenograms the complex trees have been stripped down to just a few primary and secondary branches. The explanation rests in the tradeoff between terminal process diameter and boundary effects, discussed above (see Voltage transfer from the soma).

Electrical and calcium signaling in spines

The function of spines remains a mystery. One longstanding hypothesis has been that attenuation across the spine neck sculpts the signal generated at the synapse: ". . . [the postsynaptic

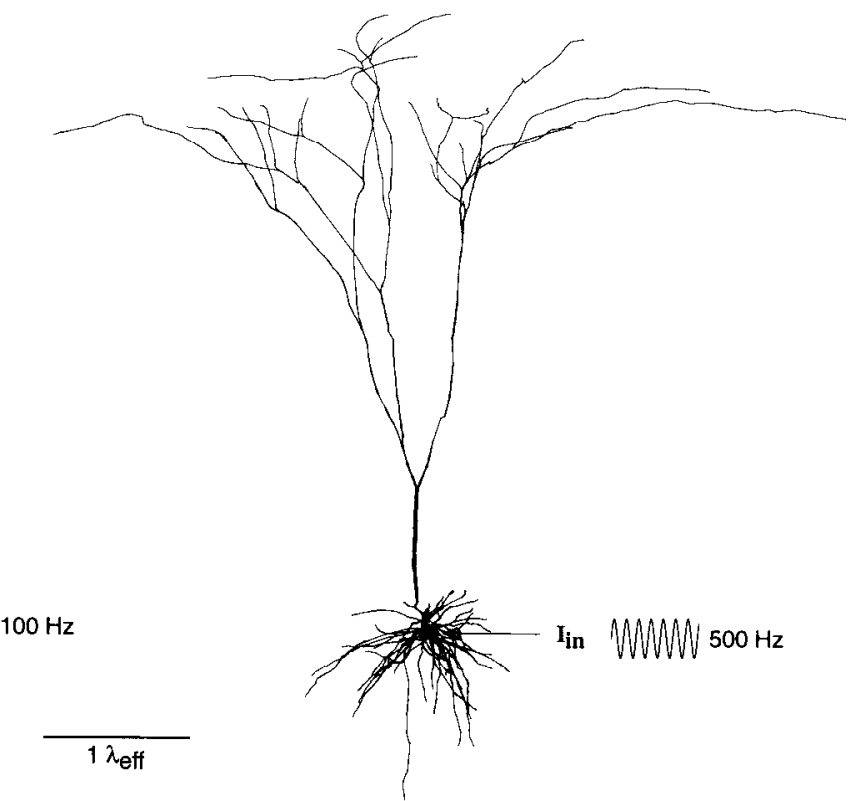

potential] must be greatly attenuated during its passage through [spine necks] which probably offer considerable ohmic resistance because of their extreme slenderness" (Chang, 1952). This idea has been analyzed and refined over the years by a number of authors who found that if the synaptic conductance is small (e.g., $<0.5 \mathrm{nS}$; see Bekkers and Stevens, 1990), then under reasonable assumptions the synapse behaves as a current source. In this case the high input resistance at the spine head causes a substantial local boost leading to substantial voltage attenuation from the spine head, but the passive electrical role of the spine in modulating synaptic efficacy is negligible (Rall and Rinzel, 1971; Rall, 1974; Koch and Poggio, 1983; Segev and Rall, 1988; reviewed in Koch and Zador, 1993).

The MET can be used to develop intuitions about the electrical role of spines. Figure $10 A$ (left) shows voltage attenograms of a model spine. In the centripetal view-log-attenuation toward the dendrite (bottom left)-the spine neck has sprouted considerably relative to the dendritic shaft, reflecting the amplification of the subsynaptic voltage relative to the dendritic; the spine head has been reduced to a sliver. But this view must be interpreted carefully. This centripetal view does not tell us the effect, or cost, of moving a synapse from the dendritic shaft to the spine head. As explained above, for this we must consider the centrifugal view (top left), which gives the cost of moving an input from one site to another. In this attenogram the entire spine has been reduced to a sliver, reflecting the efficacy of voltage transfer from the shaft to the spine head. It is this view that illustrates why a passive spine can be expected to play little

Figure 9. METs of four neuronal classes emphasize electrotonic variety. The same pyramidal cell from layer 5 of cat visual cortex considered in Figures $4-8$ is compared with a layer $2 / 3$ pyramidal cell, a cerebellar Purkinje cell from the guinea pig and a region CA1 hippocampal cell. The anatomical reconstructions are shown on the left column. Only attenuation out is considered, drawn to scale in the middle column and normalized for easier comparison in the right column. The scale representations emphasize the wide range of electrotonic compactness, from the Purkinje cell that shrinks to a point, to the CAl cell with its sprouting central stalk. The normalized representations emphasize the variable degree to which neuronal architecture is distorted by the MET, from the practically unchanged layer $2 / 3$ cell to the Purkinje and CA1 pyramidal, whose complex trees have been stripped down to just a few primary and secondary branches (their somas vanish altogether because they are essentially isopotential). For all cells, default parameters were used: $R_{m}=20 \mathrm{k} \Omega \mathrm{cm}^{2}, R_{i}=100 \Omega \mathrm{cm}$, and $C_{m}=1 \mu \mathrm{F} / \mathrm{cm}^{2}$. 


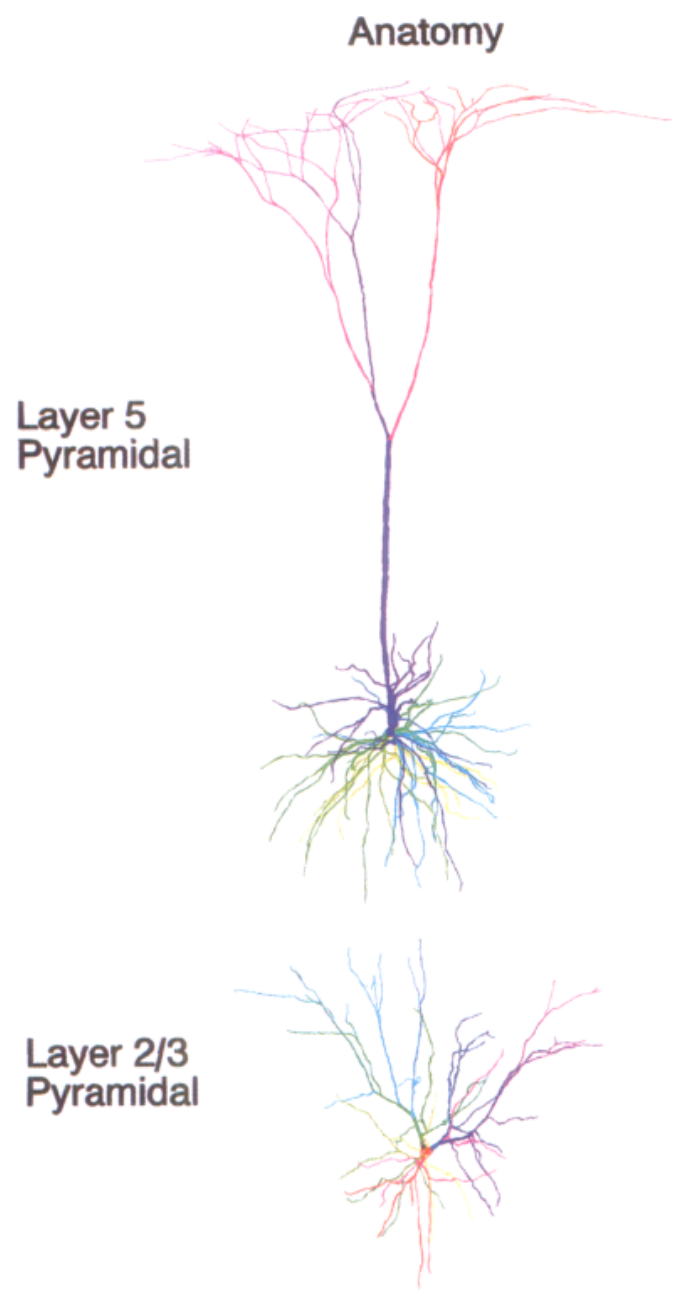

Attenuation (out)

To scale
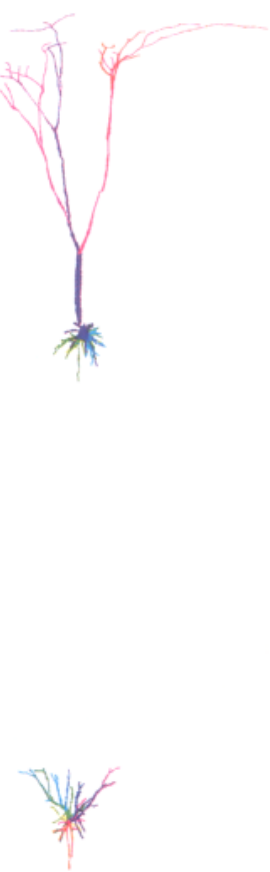

Purkinje

CA1

Pyramidal

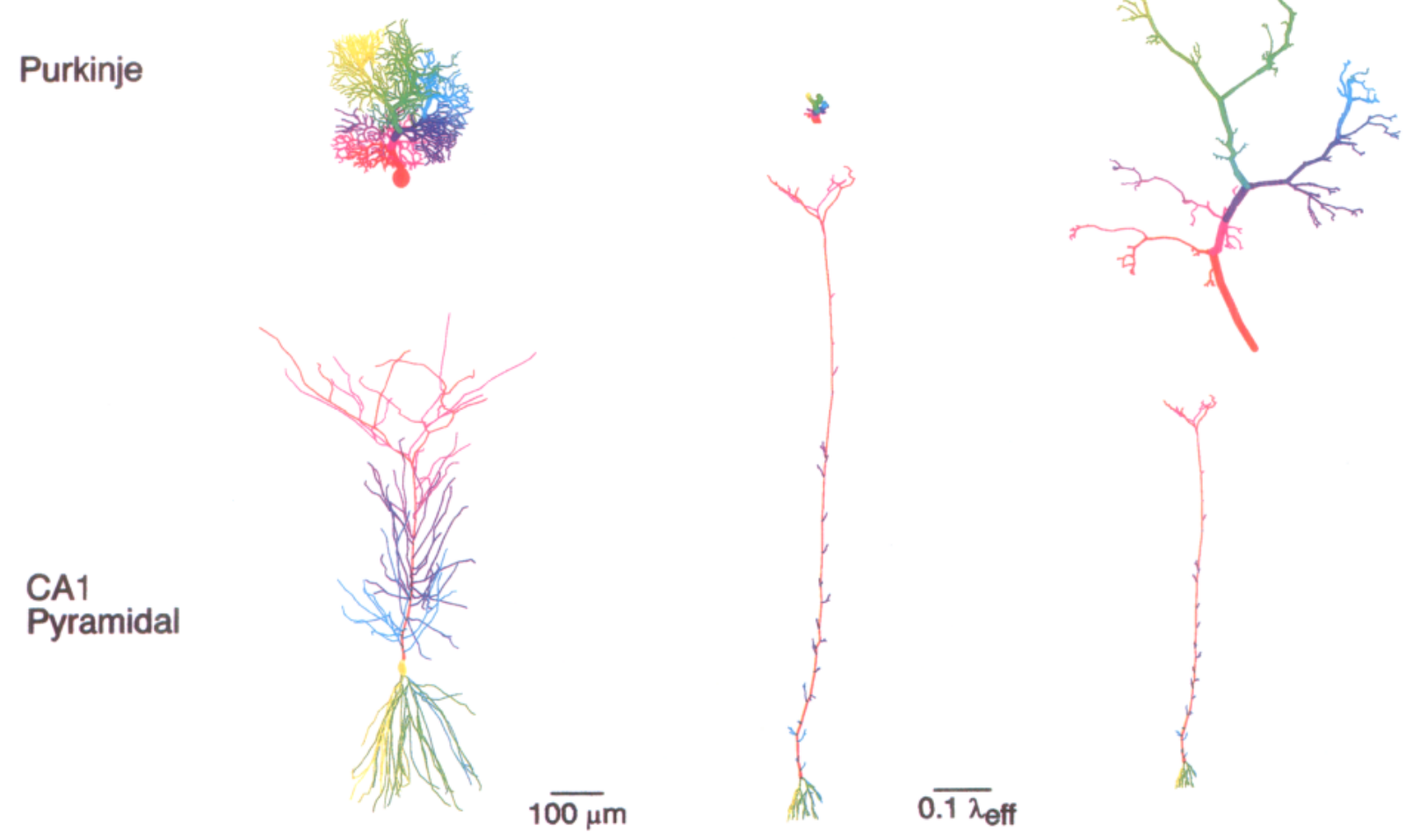

Attenuation (out)

Normalized

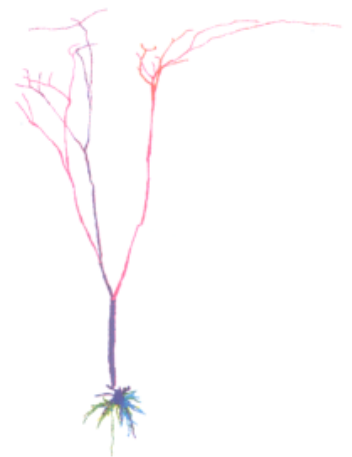


Figure 10. METs provide insight into the function of spines. A model spine was used to explore electrical $(A)$ and chemical $(B)$ signaling in spines. The model spine was the same used in $\mathrm{Za}$ dor and Koch (1994). Its anatomical dimensions (center) were: $0.05 \mu \mathrm{m}$ (neck radius), $0.25 \mu \mathrm{m}$ (head radius), $1.0 \mu \mathrm{m}$ (neck length), $0.3 \mu \mathrm{m}$ (head length). $A$, In the top METs show centrifugal transfer. The spine has been reduced to a sliver, indicating that there is essentially no cost (see text) for placing an input on the spine head rather than at the dendrite. The bottom METs show centripetal transfer. Here, the attenogram is much longer because of the boost provided by the high input resistance at the spine head, while the delayogram is longer because of $D_{i n}$ is much greater at the dendritic shaft than the spine head (at the shaft, $R_{i n}=260$ $\mathrm{M} \Omega$ and $D_{i n}=5 \mathrm{msec}$ ). $B$, In the chemical METs, both the centrifugal and the centripetal representations are of significant size. This shows that the cost of placing an chemical input at the spine head is substantial, both in terms of attenuation and delay.
A

Voltage
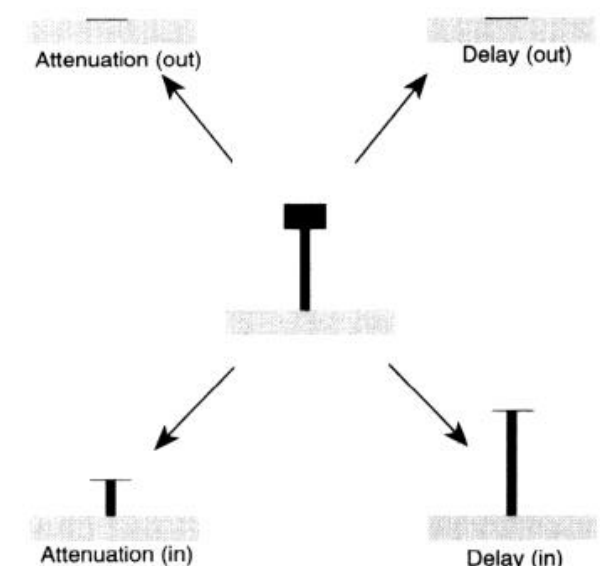

Attenuation (in)
B

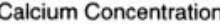

role in modulating synaptic efficacy of small inputs: there is no cost at all for moving the input from the shaft to the head.

Just the same principles apply to the propagation delay (Fig. $10 A$, right). As anticipated, the delay from the head to the neck is much longer than from the neck to the head. But if we are interested in the effect of the spine, then as before we must consider the cost, or additional delay, of moving the synapse to the spine head. In this delayogram, just as in the corresponding attenogram, the spine has shrunk to a sliver, showing that from an electrical point of view the delay due to a passive spine is negligible.

An alternative hypothesis of spine function has emerged that focuses on a possible biochemical role. As first proposed by Shepherd (1974), spines may serve to compartmentalize calcium and other second messengers (reviewed in Koch and Zador, 1993). Computer models of nonlinear spine calcium dynamics (Gamble and Koch, 1987; Holmes and Levy, 1990; Zador et al., 1990) have validated the reasonableness of this hypothesis. While direct experimental approaches have only recently become feasible, preliminary results are promising (Guthrie et al., 1991; Müller and Connor, 1991).

Recently, a linearized model of calcium dynamics in a spine has been constructed that retains the key qualitative features of the nonlinear model from which it was derived (Zador and Koch, 1994). The linearized equations that govern calcium dynamics are formally equivalent to the cable equation, so that quantities such as the chemical input and transfer resistance, and chemical attenuation can be defined. We can therefore generate morphoelectrotonic transforms to explore the effects of spines on chemical signaling (Fig. 10B).

Although the equations governing calcium and electrical signaling are identical, the parameters are so different that very different behavior emerges. As in the electrical case, attenuation in is much larger than attenuation out. But in contrast to the electrical case, both directions show substantial attenuation. While the actual magnitudes of the attenuation depend on several parame- ters-most notably the pump density in the spine neck (see Zador and Koch, 1994) - the qualitative result is valid over a wide parameter range. Similarly, the delayograms show that spines add a several millisecond delay in both directions. It is interesting that the inward delay is of comparable magnitude in both the chemical and electrical transforms, but the outward delay is much larger in the chemical transforms. These calcium attenograms vividly reinforce the idea that spines may play a much larger role in modulating chemical than electrical signaling.

\section{Discussion}

\section{Visualization of electrotonic structure}

The central contribution of this article is the development of the morphoelectrotonic transform (MET), a tool for visualizing the functional implications of electrotonic structure. The MET permits the complexities of intraneuronal signaling to be rapidly and intuitively grasped. It provides a compact representation of a tremendous quantity of data: the functional relationship of each point to every other point in the neuron. Where the efficacy and rate of signal transfer must be inferred from the morphological representation of the neuron, the MET represents these quantities directly.

The MET is not a substitute for cable theory (Rall, 1959, 1977); rather, it is an aid that makes it easier to understand. While it is true that the transformations stand on their ownthey can be interpreted without delving deeply into cable theory-we have tried here to extract the real insights that come from explaining the transformations in terms of basic principles of electrotonus. In interpreting the transformations, we are led to draw connections that we might not otherwise have made.

\section{Novel measures of electrotonic structure}

The MET is based on two novel measures of electrotonic structure, $L_{i j}$ and $P_{i j}$. The log-attenuation $L_{i j}$ is a generalization of electrotonic distance to arbitrarily branched passive neurons. In an infinite cylinder, the electrotonic distance $X$ is convenient 
because it hears a simple and direct exponential relation to the voltage at any point: $V(X)=V_{0} e^{-X}$. For each unit of $X$ from a stimulus there is an $e$-fold drop in voltage. In the case of an infinite cylinder, and in this case alone, $L_{i j}$ and $X$ are identical. In all other structures the two measures differ; only $L_{i j}$ preserves the $e$-fold relation.

$L_{i j}$ is a direct measure of the efficacy of intraneuronal signaling, and its fundamental unit is the $e$-fold drop. Specifying $L_{t}$ permits the voltage at $j$ to be expressed as a simple exponential function of the voltage $\tilde{V}_{i}$ at $i: \tilde{V}_{j}=\tilde{V}_{i} e^{-L_{i j}}$. Thus, even in a complex dendritic tree the voltage at any point can be related to the voltage at any other point by a simple exponentiation.

It the $e$-fold drop is taken as the defining feature of electrotonic distance, then $L_{i j}$ represents its most natural generalization to complex structures. The conventional measure $X$ takes the morphological definition as the defining feature: it is the physical distance $x$ scaled by the space constant $\lambda$. This generalization has the advantage of being easy to compute, but in structures more complex than an infinite cylinder the relation of $X$ to intraneuronal electrical transfer is not straightforward because the boundary conditions strongly affect signal transfer. For example, even in the simplest of structures, the finite cable of length $L$, the voltage at $X$ is given by $V(X)=V(0) \cosh (L-X) / \cosh (L)$, and in more complex structures the expressions rapidly become more cumbersome. Yet to infer the efficacy of signal transfer from $X$ requires evaluation of such expressions. Because the $\log$-attenuation $L_{i i}$ preserves the intuitively appealing exponential relationship in arbitrary branched structures, it effectively shifts the computational burden to the computer that must evaluate these expressions. As demonstrated here, the availability of fast computers and efficient algorithms makes this task easily manageable.

The second measure used here is the propagation delay $P_{i j}$, which reflects the rate at which transient signals such as synaptic impulses propagate along the dendritic tree (Agmon-Snir and Segev, 1993). $P_{i j}$ has no analogy in classical cable theory; it is an entirely new measure. $P_{i j}$ is based on the centroid of a signal, which unlike other measures such as the peak admits an elegant analytic treatment even in complex dendritic trecs. $P_{i j}$ opens up a host of questions for discussion which could not previously be broached in a rigorous fashion.

The key property of $L_{i j}$ and $P_{i j}$ that make them a suitable basis for the MET is their additivity. If $j$ is a point between $i$ and $k$, then $L_{i k}=L_{i j}+L_{j k}$ and likewise $P_{i k}=P_{i j}+P_{j k}$. In this sense they behave as true distances. Because they reflect function, they are a natural substitute for the physical distances of three-dimensional morphological rendering.

In contrast to true distances, $L_{i j}$ and $P_{i j}$ have direction. They are asymmetric, so that the distance from $i$ to $j$ is in general not equal to the distance from $j$ to $i$. This asymmetry implies that the view from each point in the neuron is unique. This asymmetry is not some unfortunate consequence of the definitions. Rather, it reflects a real and inescapable property of dendritic trees: each point bears a unique relation to every other point.

\section{Scope of the MET}

The assumption of passive dendritic membrane underlying the present analysis is unrealistic in at least two respects. First, synaptic inputs are inherently nonlinear; they are produced by a conductance change that perturbs the system. Second, there is a growing evidence that dendrites of many neuronal classes are endowed with voltagc-gated channcls (Miyakawa et al., 1992; Regehr et al., 1992; Stuart and Sakmann, 1994; reviewed in Mel,
1994). Nevertheless, any analysis of nonlinear dendritic properties must begin with a thorough understanding of the passive case (Rapp et al., 1994). Further, for many experimental studies, particularly those involving synaptic physiology, pharmacological agents are employed to linearize the membrane.

The present framework is not strictly limited to current injection; it is valid even for the localized conductance changes underlying a synaptic or action potential. As a result, neither $L_{i j}$ nor $P_{i j}$ depend on the properties of the membrane at the input point, $i$. They do depend on the properties of the membrane in the direction of signal propagation; there the membrane (and axial) properties must be independent of voltage and time for the ME"I to be valid. 'I his does not mean that, in principle, the MET can not be extended to treat nonlinear cases. However, in these cases the MET will vary in time and will depend on the input (voltage) magnitude at point $i$. The MET may become rather cumbersome to compute and will probably be hard to interpret. Finally, there remains the question of applying the MET to trees that receive multiple current inputs. The advantage of a single input considered in this article is that, in passive trees, the direction (voltage gradient) is well defined in this case. This is not the case for multiple inputs that, depending on input locations and magnitude, can create many voltage maxima and minima in the tree. Still, for any particular distribution and magnitudes of multiple inputs, the MET can be applied in a piecewise manner between the various sites of voltage maxima and minima in the tree.

\section{Applications of the MET}

We have illustrated a number of applications of the MET. First, it can be used to obtain a thorough understanding of signaling in a single neuron. In the case of the cortical pyramidal cell studied here, we considered both centrifugal and centripetal attenograms and delayograms from the soma, as well as additional attenograms from different sites and sinusoidal stimuli of different frequencies. The interpretation of these METs drew heavily upon the nine properties listed for reasoning about $L_{i j}$ and $P_{i j}$. With a few representative METs, a full picture of the electrotonic structure emerged. Parts of this picture were a surprise, at least to us, including the relative similarity between the apical and basal dendritic trees.

The MET also provides a tool for comparing neurons from different regions of the brain. For this we chose a single transform, the centrifugal somatic attenogram, but for some purposes another MET night have been preferable. Differences between these cell types were vividly depicted, both in the scale and the form. Some neurons, such as the layer $2 / 3$ cortical pyramidal cell, were almost invariant under the transformation, while others such as the Purkinje cell changed dramatically. Thus, the Purkinje cell, one of the most complex in the brain, actually appeared simpler than the layer $2 / 3$ cell. Similarly the layer 5 cell, which was physically larger than CAl pyramidal cell, actually appeared electrotonically more compact. Comparable results were obtained by Tsai et al. (1994), who extended the results of Zador (1993) on a region CA1 hippocampal pyramidal cell to a cell from region CA3 and another from the dentate gyrus.

The MET can also provide insight into the function of spines. In the case of electrical transfer, the centrifugal transforms showed that the spine changes essentially nothing about the signal at the dendritic shaft. When these transforms were applied to calcium transfer, the role of spincs appcarcd quitc different.

Other possible applications were not developed here. We have 
used the MFT to explore the effect of varying membrane parameters, either locally or globally (not shown). Such studies help understand the effect of our uncertainty about the values of certain parameters, including the membrane resistivity $R_{m}$ (Rall et al., 1992). The MET can also be applied to other aspects of voltage transfer, including the signal width, which may have important consequences for input synchronization in dendritic trees (Agmon-Snir, 1994). Moreover, the variable viewpoint intrinsic to the two-port formalism opens a broad class of problems for consideration. For example, the subsynaptic voltage determines in part whether a Hebbian synapse will be potentiated. The impedance matrix provides the foundation for understanding these effects (Pearimutter, 1994).

We conclude that the MET is a powerful tool for appreciating the functional consequences of dendrites and spines. It emphasizes how intraneuronal signaling is determined by both morphology and electrical properties, and how because of its inherent asymmetry signal transfer depends on the point of view.

\section{References}

Agmon-Snir H (1994) A novel approach to the analysis of dendritic transients. PhD thesis, Hebrew University of Jerusalem.

Agmon-Snir H, Segev I (1993) Signal delay and input synchronization in passive dendritic structures. J Neurophysiol 70:2066-2085.

Barrett J, Crill W (1974) Influence of dendritic location and membrane properties on the effectiveness of synapes on cat motoneurones. $J$ Physiol (Lond) 293:324-345.

Bekkers J, Stevens C (1990) Presynaptic mechanism for long-term potentiation in the hippocampus. Nature 346:724-729.

Brown TH, Zador AM, Mainen ZI, Claiborne BJ (1992) Hebbian computations in hippocampal dendrites and spines. In: Single neuron computation (McKenna T, Davis J, Zornetzer SF, eds). New York: Academic.

Butz E, Cowan J (1974) Transient potentials in dendritic systems of arbitrary geometry. Biophys J 14:661-689.

Carnevale N, Johnston D (1982) Electrophysiological characterization of remote chemical synapses. J Neurophysiol 47:606-621.

Chang H (1952) Cortical neurons with particular reference to the apical dendrites. Cold Spring Harbor Symp Quant Biol 17:189-202.

Douglas R, Martin K, Whitteridge D (1991) An intracellular analysis of the visual responses of neurones in cat visual cortex. J Physiol (Lond) 440:659-696.

Fleshman J, Segev I, Burke R (1988) Electrotonic architecture of typeidentified $\alpha$-motoncurons in the eat spinal cord. J Neurophysiol 60 : 60-85.

Gamble E, Koch C (1987) The dynamics of free calcium in dendritic spines in response to repetive synaptic input. Science 236:1311-1315.

Guthrie P, Segal M, Kater SB (1991) Independent regulation of calcium revealed by imaging dendritic spines. Nature 354:76-80.

Hines M (1989) A program for simulation of nerve equations with branching geometries. Int J Biomed Comput 24:55-68.

Holmes W, Levy W (1990) Insights into associative long-term potentiation from computational models of NMDA receptor-mediated calcium influx and intracellular calcium concentration changes. J Neurophysiol 63:1148-1168

Jack J, Noble A, Tsien R (1983) Electrical current flow in excitable membranes, 2nd edition. Oxford: Oxford UP.

Koch C, Poggio T (1983) A theoretical analysis of the electrical properties of spines. Proc R Soc Lond [Biol] 218:455-477.

Koch C, Poggio T (1985) A simple algorithm for solving the cable equation in dendritic trees of arbitrary geometry. $J$ Neurosci Methods $12: 303-315$

Koch C, Zador A (1993) The function of dendritic spines: devices subserving biochemical rather than electrical compartmentalization. J Neurosci 13:413-422.

Koch C, Poggio T, Torre V (1982) Retinal ganglion cells: a functional interpretation of dendritic morphology. Proc R Soc Lond [Biol] 298: 227-264.

McKenna T, Davis J, Zornetzer SF, eds (1992) Single neuron computation. New York: Academic.
Mel B (1994) Information processing in dendritic trees. Neural Comp 6 , in press.

Müller W, Connor J (1991) Dendritic spines as individual neuronal compartments for synaptic $\mathrm{Ca}^{2+}$ responses. Nature 354:73-76.

Miyakawa H, Lev-Ram V, Lasser-Ross N, Ross W (1992) Calcium transients evoked by climbing fiber and parallel fiber synaptic inputs in guinea pig cerebellar purkinje neurons. $J$ Neurophysiol 68:1178-1189.

Nitzan R, Segev I, Yarom Y (1990) Voltage behaviour along the irregular dendritic structure of morphologically and physiologically characterized vagal motoneurons in the guinea pig. J Neurophysiol 63:333-346.

Pearlmutter B (1994) Time-skew Hebb rule in a nonisopotential neuron. Neural Comp, in press.

Rall W (1959) Branching dendritic trees and motoneuron membrane resistivity. Exp Neurol 1:491-527.

Rall W (1962) Electrophysiology of a dendritic neuron model. Biophys J 2:145-167.

Rall W (1967) Distinguishing theoretical synaptic potentials computed for different somadendritic distributions of synaptic inputs. J Neurophysiol 30:1138-1168.

Rall W (1974) Dendritic spines, synaptic potentcy and neuronal plasticity. In: Cellular mechanisms subserving changes in neuronal activity (Woody C, Brown KA, Crow J, Knispel J, eds), pp 13-22. Los Angeles: University of California.

Rall W (1977) Core conductor theory and cable properties of neurons. In: Handbook of physiology, Volume 1 (Kandel E, ed), pp 39-98. Bethesda: American Physiological Society.

Rall W (1989) Cable theory for dendritic neurons. In: Methods in neuronal modeling (Koch C, Segev I, eds), pp 9-62. Cambridge, MA MIT Press.

Rall W, Rinzel J (1971) Dendritic spine function and synaptic attenuation. Soc Neurosci Abstr 1:64.

Rall W, Rinzel J (1973) Branch input resistance and steady attenuation for input to one branch of a dendritic neuron model. Biophys J 14: 648-688.

Rall W, Burke R, Holmes W, Jack J, Redman S, Segev I (1992) Matching dendritic neuron models to experimental data. Physiol Rev 72 : $159-186$.

Rapp M, Segev I, Yarom Y (1994) Physiology, morphology and detailed passive models of cerebellar purkinje cells. J Physiol (Lond), in press.

Regehr W, Konnerth A, Armstrong C (1992) Sodium action potentials in the dendrites of cerebellar purkinje cells. Proc Natl Acad Sci USA 89:5492-5496.

Rinzel J, Rall W (1974) Transient response in a dendritic neuron model for current injected at one branch. Biophys J 14:759-790.

Segev I, Rall W (1988) Computational study of an excitable dendritic spine. J Neurophysiol 60:499-523.

Segev I, Rinzel J, Shepherd G, eds (1994) The theoretical foundation of dendritic function: collected papers of Wilfrid Rall with commentaries. Cambridge, MA: MIT Press.

Shepherd G (1974) The synaptic organization of the brain, 1st edition. New York: Oxford UP.

Stuart G, Sakmann B (1994) Active propagation of somatic action potentials into neocortical pyramidal cell dendrites. Nature 367:69-72.

Torre V, Poggio T (1978) A synaptic mechanism possibly underlying directional selectivity to motion. Proc R Soc Lond [Biol] 202:409-416.

Tsai K, Carnevale N, Claiborne B, Brown T (1994) Efficient mapping from neuroantomical to electrotonic space. Network 5:21-64.

Tuckwell H (1989) Introduction to theoretical neurobiology, Vol 1. Cambridge.

Wolfram S (1992) Mathematica: a system for doing mathematics by computer, 2nd ed. Reading, MA: Addison-Wesley.

Zador A (1993) Biophysics of computation in single hippocannal neurons. $\mathrm{PhD}$ thesis, Yale University.

Zador A, Koch C (1994) Linearized models of calcium dynamics: formal equivalence to the cable equation. J Neurosci 14:4705-4715.

Zador AM, Pearlmutter BA (1993) Efficient computation of sparse elements of the inverse of a sparse near-tridiagonal matrix with application to the nerve equation. Technical Report OGI-CSE-93-003, Oregon Graduate Institute of Science \& Technology, Department of Computer Science and Engineering, Beaverton, Oregon.

Zador A, Koch C, Brown T (1990) Biophysical model of a Hebbian synapse. Proc Natl Acad Sci USA 87:6718-6722.

Zador A, Claiborne B, Brown T (1991) Electrotonic transforms of hippocampal neurons. Soc Neurosci Abstr 17:1515. 